\title{
RFID-based Indoor Positioning by Using Monte Carlo Algorithm
}

\author{
Lujie $\mathrm{Wu}^{1, \mathrm{a}}$, Gang Wang ${ }^{2, \mathrm{~b}}$ \\ ${ }^{1}$ University of Science and Technology of China (USTC) Hefei, China \\ ${ }^{2}$ Key Laboratory of Electromagnetic Space Information Chinese Academy of Sciences (CAS), Hefei, \\ China \\ awljie@mail.ustc.edu.cn, ${ }^{\mathrm{b}}$ gwang01@ustc.edu.cn
}

Keywords: Radio-frequency identification(RFID), indoor positioning, accuracy, Monte Carlo.

\begin{abstract}
An indoor positioning scheme for a moving vehicle equipped with UHF radio-frequency identification (RFID) system is proposed by using Monte Carlo-based algorithm. By evaluating and updating the random sampling positions marked by passive RFID tags on ground, Monte Carlo algorithm can be implemented successfully for positioning. Performance of the positioning algorithm has been demonstrated by both numerical and experimental results.
\end{abstract}

\section{Introduction}

RFID-based tracking systems have been considered for wireless indoor positioning for years. RFID-based positioning system usually consists of RFID reader and specially-deployed passive RFID tags on ground. By interrogating the RFID tags, recording tags responses and combing the positions of RFID tags, position of RFID reader or platform carrying RFID reader can be obtained [1]. Owing to the limited answer range of passive RFID tags, RFID-based indoor positioning scheme may provide more accurate positioning results if compared with other wireless positioning indoor schemes [2]. Since the RFID readers and RFID tags are getting cheaper and cheaper, RFID-based positioning scheme has shown great potentials.

The most common RFID-based indoor positioning scheme is constructed with a RFID reader on a moving platform connected to a single RFID reader antenna [3]. For positioning, scheme of [3] tried to range the distances between the reader antenna and RFID tags by utilizing the received signal strength indicator (RSSI). And several statistical algorithms based on RSSI have been proposed by eliminating or decreasing the interference or noise from the indoor environment [4,5]. Unfortunately, the RSSI may be severely distorted by the poor indoor wireless propagation due to interference from different obstacles. Moreover, measuring RSSI usually requires specially designed RFID reader.

In this paper, we propose an RFID-based indoor positioning scheme by using ordinary RFID reader. Based on the position information of RFID tags, by using Monte Carlo algorithm, positioning or tracking of a moving vehicle equipped with a RFID reader can be implemented. The scheme doesn't need to measure RSSI and may avoid many problems in practical indoor environment.

\section{Monte Carlo-based Positioning Algorithm}

The model for RFID-based indoor positioning is shwon in Fig. 1. On the ground, UHF passive tags are arranged in a designeted pattern, and the data of tag's coordinates has been stored in the RFID system. The interrogation zone of RFID antenna on the ground, referred to as footprint, is shown in Fig. 2. RFID tags within the footprint can be recognized by the RFID reader. Their coordinates can be acquired by checking teh deployment pattern, which can be used for vehicle positioning.

For positioning, Monte Carlo algorithm can be inplemented in two phases: prediction phase and update phase, which are given as follows.

I. Prediction Phase. At time $k$, the antenna can detect some tags whose number is set ' $n$ ' and the system can get the position coordinates of these tags. So we can estimate the position of the footprint center of the antenna preliminarily by Eq. 1 . 


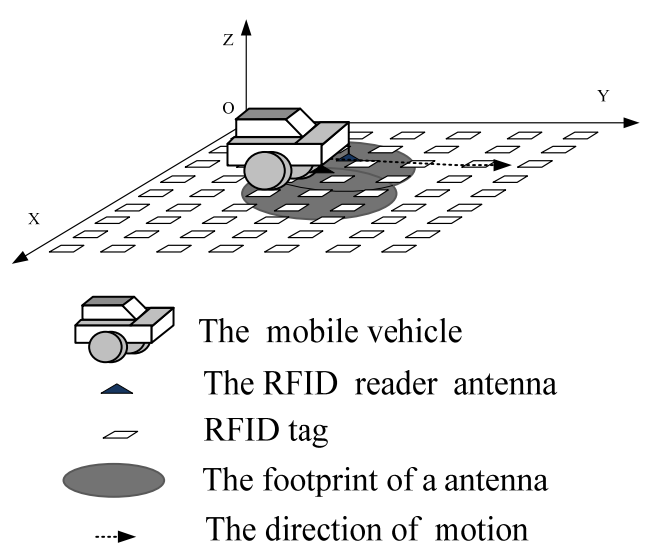

Fig. 1. RFID-based indoor positioning system.

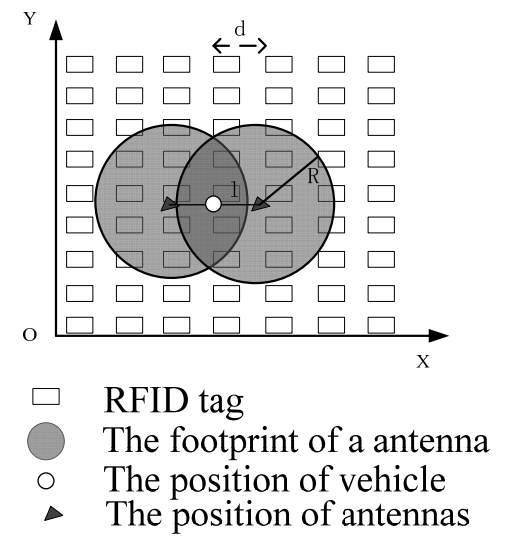

Fig. 2. Antenna footprint in moving.

$$
\left(a x_{k}^{\prime}, a y_{k}^{\prime}\right)=\left(\frac{x_{1}+x_{2}+\ldots+x_{n}}{n}, \frac{y_{1}+y_{2}+\ldots+y_{n}}{n}\right) .
$$

Where $\left(a x_{k}^{\prime}, a y_{k}^{\prime}\right)$ is the estimated footprint center position and $\left(x_{i}, y_{i}\right)$ is the position of the detected tag ith $(i=1 \ldots n)$.

The footprint center $o_{k}$ is defined as an arbitrary probability density function within the footprint near the position $\left(a x_{k}^{\prime}, a y_{k}^{\prime}\right)$. The probability coordinates of the footprint center are formed by $N$ particles. Each particle is composed of the position hypothesis $s_{j}^{k}=\left(s x_{j}, s y_{j}\right)(j=1 \ldots N)$ of the antenna and the weight of the $j$ th particle $p(j) . S_{j}=\left\{\left(s_{j}^{k}, p(j)\right) \mid j=1,2, \cdots, N\right\}$ is the set of the sampling particles at time $k$.

II. Update Phase. At time $k$, some particles can't meet the condition that if the footprint center is on the positions of these particles, the antenna can recognize the tags which are detected in fact. So for each particle like this, we find other two particles which meet the condition, take an average of the coordinates of the two particles and use the average to replace the particle position which doesn't meet the condition.

At time $k+1$, the antenna has detected some other tags and has traveled a certain distance from time $k$. The particles at time $k$ would travel with the vehicle in the same direction and the same distance, then reach the new positions at time $k+1$. Then we analyze if each new particle can meet the condition. If the particle can't meet the condition, we give up the particle at time $k$. Then we use the Gaussian probability model to get the weight of the remained particles described by Eq. 2 .

$$
p(j)=p\left(d_{j} \mid l_{k-1}\right)=\frac{1}{\sigma \sqrt{2 \pi}} e^{\frac{\left(d_{j}-l_{k-1}\right)^{2}}{2 \sigma^{2}}} .
$$

Where $d_{j}$ is the distance between the ith particle at time $k . l_{k-1}$ is the distance of the antenna from time $k-1$ to time $k . \sigma$ is measured value variance.

By normalizing the weight of the remained particles in the set $S_{k}$, calculating the position of the footprint center $o_{k}^{\prime}$ at time $k$ by Eq. 3 .

$$
o_{k}^{\prime}=\sum_{j=1}^{M} p(j) \cdot s_{j} \quad(M \leq N) .
$$

The position of the vehicle at time $k$ is marked by averaging the positions of the two footprint centers. 


\section{Simulation and Experiment Verification}

In order to verify the performance of the proposed Monte Carlo-based positioning algorithm, simulation and experiment have been carried out. For comparison, positioning algorithm proposed in [6] is also used for our simulation and experiment data. The algorithm reported in [6] has been demonstrated to provide high positioning precision.

The positioning performance can be demonstrated by presenting localization error during moving and positioning, which is defined as Eq. 4.

$$
\text { Error distance }=\sqrt{\left(x_{k}-x_{k}^{\prime}\right)^{2}+\left(y_{k}-y_{k}^{\prime}\right)^{2}} \text {. }
$$

Where $\left(x_{k}, y_{k}\right)$ is the real coordinates of vehicle and $\left(x_{k}^{\prime}, y_{k}^{\prime}\right)$ is the estimated coordinates of the vehicle by the positioning algorithm.

The tag deployment pattern on the ground is listed in Table 1.

TABLE 1. TAG DePloyment PATTERN

\begin{tabular}{lc}
\hline \hline \multicolumn{1}{c}{ Parameter list } & Value \\
\hline interval between tags & $50 \mathrm{~cm}$ \\
number of total tags & 70 \\
number of sampling particles & 300 \\
average error of distance & $10 \%$ \\
\hline \hline
\end{tabular}

Same as in [6], we set an antenna footprint of radius of $90 \mathrm{~cm}$ on the ground. For tag deployment pattern in Table 1, positioning results with the proposed Monte Carlo-based algorithm and algorithm in [6] are shown in Fig. 3.
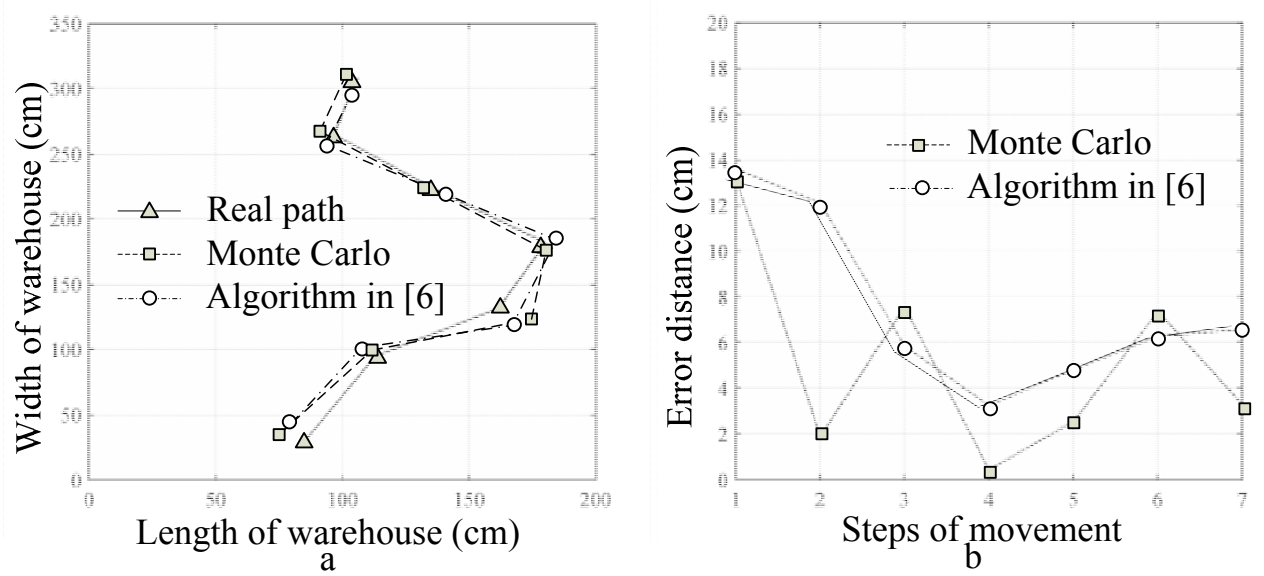

Fig. 3. Positioning results (a) and errors (b) with simulation data for two algorithms.

From Fig. 3, it is observed that the accuracy of Monte Carlo-based algorithm is higher than that proposed in [6]. During the moving and positioning, the average error of Monte Carlo-based algorithm is $8.89 \mathrm{~cm}$, while that of the algorithm in [6] is $10.83 \mathrm{~cm}$. The improvement may be due to the fact that the samplings are updated twice in Monte Carlo algorithm so that the effective sampling area in the Monte Carlo-based positioning are enlarged.

Experiment test is also carried out for verification. The positioning is on floor of area of $5 \mathrm{~m} \times 7 \mathrm{~m}$. A moving platform carrying an Alien RFID reader (ALR 9900) and UHF circularly-polarized antenna (gain of $8 \mathrm{dBi}$ ) are used. The antenna is carefully adjusted so that its footprint radius on the floor is $90 \mathrm{~cm}$. RFID tags on floor are deployed in a pattern defined in Table 1.

Positioning results with test data by using the proposed Monte Carlo-based algorithm and algorithm in [6] are shown in Fig. 4. 

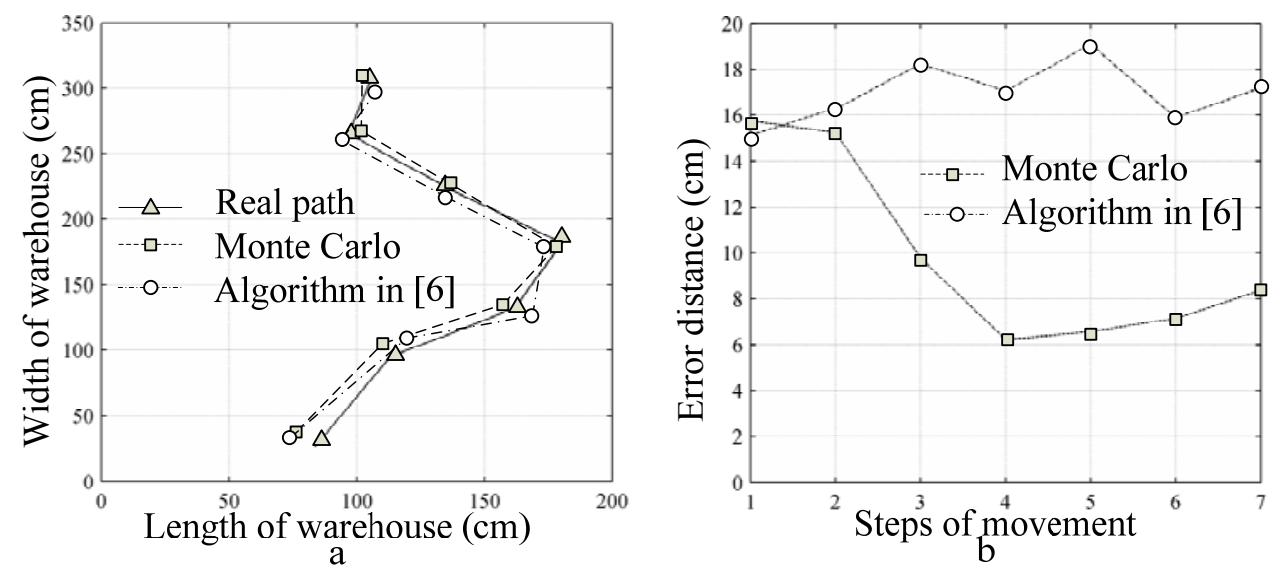

Fig. 4. Positioning results (a) and errors (b) with test data for two algorithms.

From Fig. 4, we find again that the Monte Carlo-based positioning provides better precision. By changing the tag deployment pattern, more tests have been performed. Monte Carlo-based algorithm is shown to be capable of providing higher precision.

\section{Conclusions}

For RFID indoor positioning with auxiliary passive UHF tags deployed on ground, Monte Carlo-based positioning is shown to be an effective and high precision method. In addition, the Monte Carlo-based positioning scheme can be implemented without any modification to the conventional RFID reader. It is thus a good candidate for RFID indoor positioning.

\section{Acknowledgements}

This work was supported in part by the National Natural Science Foundation of China under Grant 61272471

\section{References}

[1] M.L. Ni, Dian Zhang and M.R. Souryal: IEEE Wireless Commum. Vol. 18 (2011), p. 45-51.

[2] R. Tesoriero, J. Gallud, M. Lozano and V. Penichet: IEEE Trans. Consum. Electron. Vol. 54 (2008), p. 578-583.

[3] S. Han, H. Lim and J. Lee: IEEE Trans. Ind. Electron. Vol. 54 (2007), p. 3362-3369.

[4] E. DiGiampaolo and F. Martinelli: IEEE Trans. Ind. Electron. Vol. 59 (2012), p. 3961-3970.

[5] S.S. Saad and Z.S. Nakad: IEEE Trans. Ind. Electron. Vol. 58 (2010), p. 1961-1970.

[6] S. Park and H. Lee: IEEE Trans. Ind. Electron. Vol. 60 (2013), p. 226-234. 